This item was submitted to Loughborough's Research Repository by the author.

Items in Figshare are protected by copyright, with all rights reserved, unless otherwise indicated.

\title{
Automatic wrinkle detection using hybrid hessian filter
}

PLEASE CITE THE PUBLISHED VERSION

http://dx.doi.org/10.1007/978-3-319-16811-1_40

\section{PUBLISHER}

(c) Springer International Publishing, Switzerland

\section{VERSION}

AM (Accepted Manuscript)

\section{PUBLISHER STATEMENT}

This work is made available according to the conditions of the Creative Commons Attribution-NonCommercialNoDerivatives 4.0 International (CC BY-NC-ND 4.0) licence. Full details of this licence are available at: https://creativecommons.org/licenses/by-nc-nd/4.0/

\section{LICENCE}

CC BY-NC-ND 4.0

\section{REPOSITORY RECORD}

$\mathrm{Ng}$, Choon-Ching, Moi Hoon Yap, Nicholas Costen, and Baihua Li. 2019. "Automatic Wrinkle Detection Using Hybrid Hessian Filter". figshare. https://hdl.handle.net/2134/20252. 


\title{
Automatic Wrinkle Detection using Hybrid Hessian Filter
}

\author{
Choon-Ching Ng, Moi Hoon Yap, Nicholas Costen and Baihua Li \\ School of Computing, Mathematics \& Digital Technology \\ Manchester Metropolitan University, United Kingdom
}

\begin{abstract}
Aging as a natural phenomenon affects different parts of the human body under the influence of various biological and environmental factors. The most pronounced changes that occur on the face is the appearance of wrinkles, which are the focus of this research. Accurate wrinkle detection is an important task in face analysis. Some have been proposed in the literature, but the poor localization limits the performance of wrinkle detection. It will lead to false wrinkle detection and consequently affect the processes such as age estimation and clinician score assessment. Therefore, we propose a hybrid Hessian filter (HHF) to cope with the identified problem. HHF is composed of the directional gradient and Hessian matrix. The proposed filter is conceptually simple, however, it significantly increases the true wrinkle localization when compared with the conventional methods. In the experimental setup, three coders have been instructed to annotate the wrinkle of 2D forehead image manually. The inter-reliability among three coders is $93 \%$ of Jaccard similarity index (JSI). In comparison to the state-of-the-art Cula method (CLM) and Frangi filter, HHF yielded the best result with a mean JSI of $75.67 \%$. We noticed that the proposed method is capable of detecting the medium to coarse wrinkle but not the fine wrinkle. Although there is a gap between human annotation and automated detection, this work demonstrates that $\mathrm{HHF}$ is a remarkably strong filter for wrinkle detection. From the experimental results, we believe that our findings are notable in terms of the JSI.
\end{abstract}

\section{Introduction}

Quantitative assessment of skin condition has been an area of intense activity. There is a great interest in supplementing the dermatologist's diagnostic visual assessment of skin with objective measures [1]. These techniques are also valuable for the efficient development of effective pharmaceutical treatments. Many skin assessments have been developed over the past few years. For example, analysis of the skin surface around pores on the face [2], evaluation of facial wrinkle development over the lifetime [3], assessing facial wrinkles using automatic detection and quantification method [4]. Most of these assessments were based on clinician perspective (subjective assessment) instead of computer vision (objective assessment). Judgements are typically made on neutral-expression images. Clinician perspective focuses on the level of wrinkle severity which is assessed 

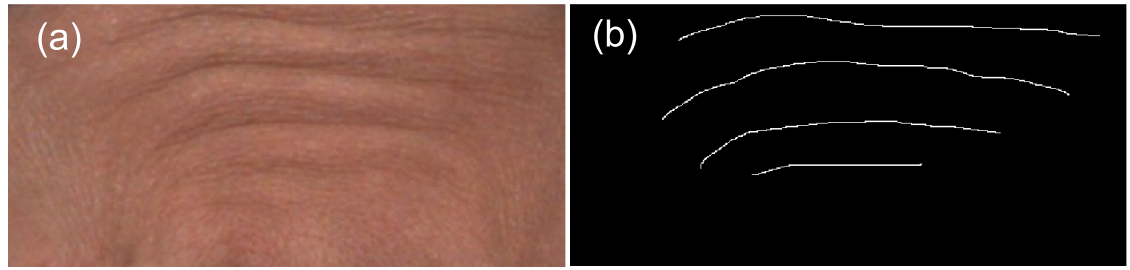

Fig. 1. Result of manual annotation. (a) and (b) present the original forehead image and the corresponding mask of manual annotation, respectively.

using either descriptive or photographically-calibrated scales, but in computer vision, the concern is on how a wrinkle is located correctly against the ground truth. The well-known shortcomings of subjective assessment limit the scientific study of treatment and environmental effects on skin aging. Therefore, this work may result as an additional tool for them.

Assume a 2D forehead image consists of four observed wrinkles as shown in Fig. 1, but an automated method might estimate less or more than that, it will lead to false detection. This will highly influence the processes such as age estimation and clinician score assessment. Cula et al. [4] claimed that an automatic facial wrinkle detection method (hereinafter referred to as CLM) has been developed with the advantage of high correlation between clinician score and the computed wrinkle index (WI). Although CLM sounds promising, further quantification is needed to localize the wrinkle and distinguish it from image noise such as illumination, hair or scar. Therefore, in this work, a novel method is highlighted on how to detect the wrinkle accurately by assessing how closely a wrinkle is located through the Jaccard Similarity Index (JSI).

The rest of this paper is organized as follows. Section 2 reviews the state-ofthe-art methods of wrinkle detection. Section 3 presents the proposed method HHF. Section 4 depicts the experimental results, and Section 5 concludes the paper.

\section{Related Works}

Automated detection of wrinkles in 2D images is an important step in age estimation [5] and synthesis [6, 7]. Aznar-Casanova et al. [8] investigated the influence of wrinkles on facial age judgments. Their results indicated that the number of wrinkles and the depth of furrows are highly correlated with the perceived facial age. Choi et al. [9] explored the accurate wrinkle representation scheme for skin age estimation. The proposed scheme shows that it may be possible to estimate skin aging automatically from skin images. Huang et al. [10] presented a robust facial expression recognition method with skin wrinkles. The side-view profile plus skin wrinkles can correctly differentiate the expressions however acted facial expressions is used instead of spontaneous expression under complex illumination condition. Bando et al. [11] proposed a simple method for 
modelling wrinkles on human skin. They demonstrated the ability of the proposed method to model realistic wrinkle shapes by comparing them with real wrinkles. Despite the fact that it is a simple method to easily model wrinkles on human skin, but this work is lack of validation against the modelled wrinkles. Batool et al. [12] investigated the forehead wrinkles as curve patterns for their discriminative power as a soft biometric. Several metrics based on Hausdorff distance and curve-to-curve correspondences are introduced to quantify the similarity. However, the information of relative positions of the curves within the pattern was not included in the experiment.

Wrinkle detection is basically a line or ridge detection problem. Few methods have been proposed in the literature. A class of popular approaches to wrinkle detection is snake-based method which use the active contour map to initialize and localize the wrinkles $[5,9,12]$. Kwon and Lobo $[13,5]$ computed wrinkles from face images to separate young adults from senior adults. The wrinkles were computed in several regions, such as on the forehead, next to the eyes, and near the cheek bones. The presence of wrinkles in a region is based on the detection of curves in that region. However, the random initialization and multiple thresholds resulted in implementation difficulty. Filtering-based method exploits the local orientation and optimizes the response to ridge-like structures. Hayashi et al. [14] utilized the edge detection method and Digital Template Hough Transform (DTHT) to extract both shorter and longer wrinkles on the face. This method is not reliable because wrinkle detection is not a boundary detection problem. Among the various wrinkle detection methods, the CLM is representative and simple. Cula et al. [4] explored the first-order derivative and Gabor filter for detecting the wrinkle length and depth, respectively. The estimation is based on the orientation and frequency of elongated spatial features. They defined the wrinkle index (WI) as the product of both wrinkle length and wrinkle depth. Wrinkle depth is derived from the Gabor filter responses. The authors claimed that the WI is highly correlated between clinical scores and outputs of CLM. However, from computer vision perspective, this work is deficient in detailed quantification of line segment localization. Therefore, this work is carried out to validate the detected lines in a $2 \mathrm{D}$ forehead image.

Frangi et al. [15] proposed a vessel enhancement filter as Frangi filter (FRF) for extracting the vessel and the Magnetic Resonance Angiography (MRA) dataset of cerebral vasculature. It has been widely used in retinal vessel detection $[16,17]$, but not in wrinkle detection. Since both wrinkle detection and vessel enhancement present similar line patterns as shown in Fig. 1, it would be interesting to find out the performance of FRF on wrinkle detection. The idea of FRF is based on second-order partial derivatives for ridge detection. Eigenvalues of Hessian matrix are utilized to extract the principal directions in which the local second-order structure of the image can be decomposed. Although both vessel and wrinkle present similar curve patterns, the underlying image quality is different. The vessel image was captured by the TopCon TRV-50 fundus camera at a $35^{\circ}$ field of view (FOV), which were digitized with 24-bit grayscale resolution and a spatial resolution of $700 \times 605$ pixels. In contrast, the original 
image of wrinkles were acquired with a normal light camera with the image resolution of $1600 \times 1200$. Higher resolution image contains more noise. Therefore, the proposed method has to deal with the pepper noise and illumination without damaging the region of interest. In addition, a retinal scan involves using a lowintensity light source through an optical coupler to scan the unique patterns of the retina [18]. Retinal scanning can be quite accurate but does not require the user to look into a receptacle and focus on a given point. As a result, these images present constant intensity across the dataset. In contrast to retinal vessels images, skin images present different challenges. Skin surface consists of pore, hair and pigmentation signal, among these attributes wrinkles present significant variations such as curve pattern, length, thickness and orientation with varied waviness and roughness.

CLM employs the local dominant orientation of first-order derivative for detecting the peak responses, it does not recognize a distinction between wrinkle and noise. Since FRF is capable on determining locally the likelihood that a ridge is present, this problem can be minimized by looking at the maximum image intensity in the direction of maximal curvature through directional gradient of image, and hence the wrinkle location can be correctly located. Therefore, a novel method namely hybrid Hessian filter (HHF) is proposed in this work. The following sections will describe the method in detail.

\section{Hybrid Hessian Filter}

Wrinkles are considered as stochastic spatial arrangements of line segment sequences, reasonably similar with those in retinal blood vessels. Wrinkle should not be confused with edge. Edge is the border between two areas while wrinkle is a line that is either darker or lighter than their neighbourhood. Therefore, edge detection methods such as Canny and Sobel are not suitable for wrinkle detection because it will produce wrinkle boundaries, not the wrinkle. In this work, we explored the multiscale second order local structure of an image. A measure of ridge-likeliness is obtained on the basis of all eigenvalues of the Hessian matrix. The eigenvalues of the Hessian matrix evaluated at each point quantify the rate of change of the gradient field in various directions. The eigenvalues are independent vector measures by the components of the second derivatives of the field at each point $(x, y)$. A small eigenvalue indicates a low change rate of the field in the corresponding eigen-direction, and vice versa [15].

Fig. 2 shows the proposed HHF for wrinkle detection. A 2D forehead image $I(x, y)$ is illustrated in Fig. 2(a) and is converted into grayscale as shown in Fig. 2(b). The gradient of $I$ is derived as Eq. (1).

$$
\nabla I(x, y)=\left(\frac{\partial I}{\partial x}, \frac{\partial I}{\partial y}\right)
$$

where $\frac{\partial I}{\partial x}$ and and $\frac{\partial I}{\partial y}$ are the directional gradient as shown in Fig. 2(c). $\frac{\partial I}{\partial y}$ emphasizes the horizontal line. Let $\frac{\partial I}{\partial y}$ denoted as $\mathcal{I}$, the Hessian matrix $\mathcal{H}$ of $\mathcal{I}$ at scale $\sigma$ is defined as Eq. (2). 
(a) Original image

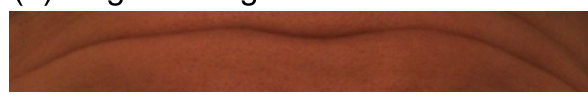

(b) Grayscale image

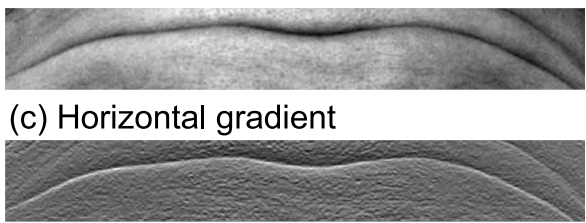

(d) Frangi filter (e) Ridge likeliness

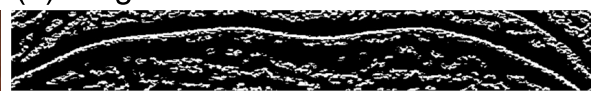

(f) Thresholding

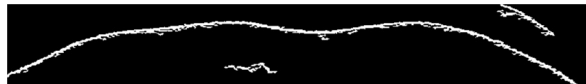

(g) Overlapping
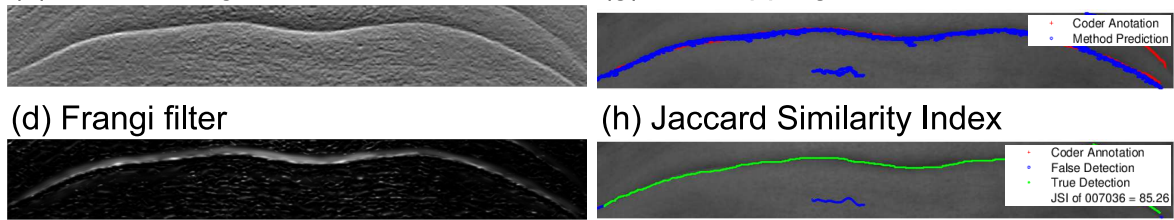

(h) Jaccard Similarity Index

Fig. 2. Hybrid Hessian filter. (a) Original image was cropped from the Bosphorus dataset [19]. (b) Colour image was converted into grayscale image. (c) Gaussian filter was used to derive the directional gradient from grayscale image. (d) FRF was applied on directional gradient image to approximate the structure around each pixel at certain scale. (e) Image vectors less than zero was preserved as ridge-like pattern. (f) Ridge image was thresholded in a degree that only wrinkle-like patterns are extracted. $(\mathrm{g})$ The overlapping between coder annotation (red line) and method estimation (blue line). (h) JSI was calculated based on the intersection area only (green line).

$$
\mathcal{H}(x, y, \sigma)=\left[\begin{array}{ll}
\frac{\partial^{2} \mathcal{I}(x, y)}{\partial \mathcal{I}(x) \partial \mathcal{I}(x)} & \frac{\partial^{2} \mathcal{I}(x, y)}{\partial \mathcal{I}(x) \partial \mathcal{I}(y)} \\
\frac{\partial^{2} \mathcal{I}(x, y)}{\partial \mathcal{I}(x) \partial \mathcal{I}(y)} & \frac{\partial^{2} \mathcal{I}(x, y)}{\partial \mathcal{I}(y) \partial \mathcal{I}(y)}
\end{array}\right]=\left[\begin{array}{ll}
\mathcal{H}_{a} & \mathcal{H}_{b} \\
\mathcal{H}_{b} & \mathcal{H}_{c}
\end{array}\right]
$$

where $\mathcal{H}_{a}, \mathcal{H}_{b}$ and $\mathcal{H}_{c}$ are the outputs of second derivative [15]. Each of the approximations is the convolution between $\mathcal{I}$ and Gaussian kernels $g_{1}(\sigma), g_{2}(\sigma)$. They are defined as Eq. (3), Eq. (4) and Eq. (5).

$$
\begin{aligned}
\mathcal{H}_{a}(x, y, \sigma) & =\mathcal{I}(x, y) * g_{1}(\sigma) \\
\mathcal{H}_{b}(x, y, \sigma) & =\mathcal{I}(x, y) * g_{2}(\sigma) \\
\mathcal{H}_{c} & =\left[\mathcal{H}_{a}\right]^{T}
\end{aligned}
$$

where $\mathcal{H}_{c}$ is the transpose of $\mathcal{H}_{a}$. The second derivative of a Gaussian kernel at scale $\sigma$ generates a probe kernel that measures the contrast between the regions inside and outside the range in the direction of the derivative. $g_{1}(\sigma)$ and $g_{2}(\sigma)$ are defined as Eq. (6) and Eq. (7).

$$
g_{1}(\sigma)=\frac{1}{2 \pi \sigma^{4}}\left[\frac{(\chi)^{2}}{\sigma^{2}}-1\right] e^{\frac{-\left(\chi^{2}+\gamma^{2}\right)}{2 \sigma^{2}}}
$$




$$
g_{2}(\sigma)=\frac{1}{2 \pi \sigma^{6}}(\chi \gamma) e^{\frac{-\left(\chi^{2}+\gamma^{2}\right)}{2 \sigma^{2}}}
$$

$\chi$ and $\gamma$ are Gaussian kernels with vertical and horizontal direction and defined as Eq. (8) and Eq. (9).

$$
\begin{array}{ll}
\chi=\sum_{i=-3 \sigma}^{3 \sigma} \sum_{j=-3 \sigma}^{3 \sigma}\left(k_{i, j} \times i\right) \quad, \mathbf{k}=1 \\
\gamma=\sum_{i=-3 \sigma}^{3 \sigma} \sum_{j=-3 \sigma}^{3 \sigma}\left(k_{i, j} \times j\right) \quad, \mathbf{k}=1
\end{array}
$$

In order to extract the eigen-direction in which a local structure of the image is decomposed, eigenvalues $\lambda_{1}$ and $\lambda_{2}$ of the Hessian matrix are defined as Eq. (10) and Eq. (11).

$$
\begin{aligned}
& \lambda_{1}(x, y, \sigma)=\frac{1}{2}\left[\mathcal{H}_{a}+\mathcal{H}_{c}+\left(\sqrt{\left(\mathcal{H}_{a}-\mathcal{H}_{c}\right)^{2}+4 \mathcal{H}_{b}^{2}}\right)\right] \\
& \lambda_{2}(x, y, \sigma)=\frac{1}{2}\left[\mathcal{H}_{a}+\mathcal{H}_{c}-\left(\sqrt{\left(\mathcal{H}_{a}-\mathcal{H}_{c}\right)^{2}+4 \mathcal{H}_{b}^{2}}\right)\right]
\end{aligned}
$$

The similarity measures $\mathcal{R}$ and $\mathcal{S}$ are defined as Eq. (12) and Eq. (13).

$$
\begin{aligned}
& \mathcal{R}(x, y, \sigma)=\left(\frac{\lambda_{1}}{\lambda_{2}}\right)^{2} \\
& \mathcal{S}(x, y, \sigma)=\lambda_{1}^{2}+\lambda_{2}^{2}
\end{aligned}
$$

where $\lambda_{2}$ is set to eps $\approx 2^{(-52)}$ if $\lambda_{2}==0$. This is to avoid the $\lambda_{1}$ divided by zero. The curvilinear likeliness measure $\mathcal{E}$ is defined as Eq. (14).

$$
\mathcal{E}(x, y, \sigma)=\left\{\begin{array}{c}
0 \\
\text { if } \lambda_{2}<0 \\
\mathrm{e}^{-\frac{\mathcal{R}}{2 \beta_{1}^{2}}}\left[1-\mathrm{e}^{-\frac{\mathcal{S}}{2 \beta_{2}^{2}}}\right] \text { otherwise }
\end{array}\right.
$$

In this work, we are interested the dark area which represents the wrinkles. The sign of $\lambda_{2}$ indicates a pixel belongs to bright (negative) or dark (positive). Therefore, $\lambda_{2}<0$ highlights the data of interest and discards the noisy patterns [15]. Due to the ridge is analyzed at different scales $\sigma$, the response of the filter will be maximum at a scale that approximately matches the size of the ridge to detect as given in Eq. (15) where $\sigma_{\min }$ and $\sigma_{\max }$ are the minimum and maximum scales at which relevant structure are expected to be found (as shown in Fig. 2(d)).

$$
\mathcal{L}(x, y)=\max _{\sigma_{\min } \leqslant \sigma \leqslant \sigma_{\max }}[\mathcal{E}(x, y, \sigma)]
$$


Once the ridge is derived from the FRF, the ridge-like pattern is preserved and the initial wrinkle mask is generated as shown in Fig. 2(e). The ridge likeliness is defined as Eq. (16).

$$
\mathcal{L}(x, y)=\left\{\begin{array}{cc}
0 \text { if } \mathcal{L}(x, y)>0 \\
1 & \text { otherwise }
\end{array}\right.
$$

Next, each region of interest (8-connected pixels) is filtered by an area threshold where regions less than 250 pixels are removed and the output is the estimated forehead wrinkle as shown in Fig. 2(f). Note that the area threshold is based on the initial image resolution.

In this work, we set the kernel scale $\sigma$ to $1,3,5,7 ; \beta_{1}$ controls the sensitivity of the filter to the measure $\mathcal{R}$ and the default value is $0.5 ; \beta_{2}$ depends on the greyscale range of the ridge of interest and controls the sensitivity of the filter to the measure $\mathcal{S}$ and the default value is 15 (refer to [15]).

Fig. 2(g) illustrated the overlapping between manual annotation and predicted wrinkle of the HHF. A polynomial fitting is implemented on the predicted wrinkle to localize the center line of the wrinkle and it is used for calculating the JSI denoted as Eq. (17). In computer vision, we are interested in how each method estimates correctly a line/segment. In this context, we used the JSI [20] to measure the reliability of wrinkle detection method. The Jaccard index $\mathfrak{J}$ is calculated by the intersection of $\mathfrak{A}$ and $\mathfrak{B}$ divided by the union of $\mathfrak{A}$ and $\mathfrak{B} . \mathfrak{A}$ and $\mathfrak{B}$ are annotations of two different coders, respectively.

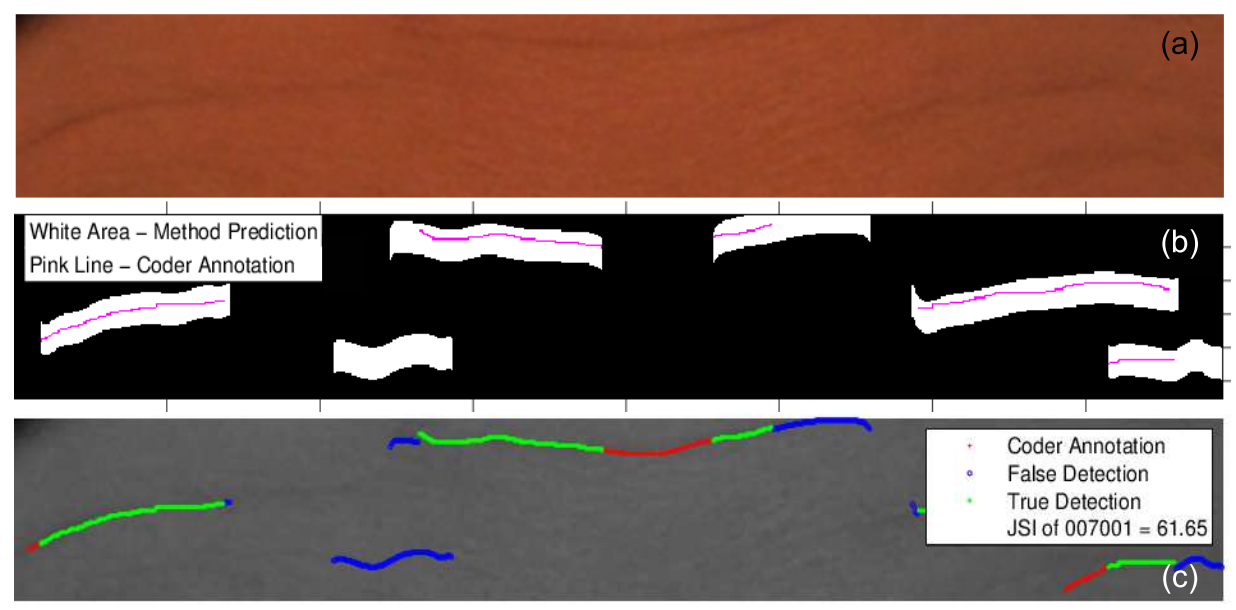

Fig. 3. Pixel overlapping between coders. (a) Original image. (b) Method annotation (or white area) has expanded nine pixels to top and bottom from its original location as shown in (c) as blue and green line. Pink line represents the coder's annotation. (c) Blue line represents the false detection and green line shows the true prediction. The image JSI is $61.65 \%$. 


$$
\mathfrak{J}(\mathfrak{A}, \mathfrak{B})=\frac{|\mathfrak{A} \cap \mathfrak{B}|}{|\mathfrak{A} \cup \mathfrak{B}|}
$$

Fig. 2(h) shows how the JSI is calculated, red represents the manual annotation or ground truth, blue means the false detection and green is the true detection. In this work, we utilized the expansion threshold of nine pixels when calculating the JSI. From the experiment, we noticed that nine pixels produced the best overlapping in between the ground truth and estimated line. Small number of pixels will fail to hit the estimated line and large number of pixels will bias the result due to certain wrinkles are close to each other. Fig. 3 illustrates an example of how the method annotation is expanded with nine pixels to top and bottom from its original location. The white area in Fig. 3(b) is an expansion of the method prediction and it is represented by the blue and green lines in Fig. 3(c).

\section{Results and Discussion}

In order to assess the performance of the wrinkle detection algorithm, images were selected from the Bosphorus dataset [19]. This dataset consists of 106 subjects and the 2D face images were acquired under good illumination conditions with a normal light camera. According to Batool and Chellappa [12], it is easier to generate the ground truth by hand labelling and wrinkles are more obvious on the forehead in most of the images. Therefore, we repeated the same procedure by manually cropping the forehead images from the Bosphorus dataset. For each image, the forehead was manually cropped with a rectangle selector as shown in Fig. 4. Rectangle size varied from one to another. Resizing images was not included because the manual cropping was considered sufficient unless the wrinkle length and width are needed. In total we collected 100 random forehead images from the Bosphorus dataset and each forehead image is annotated

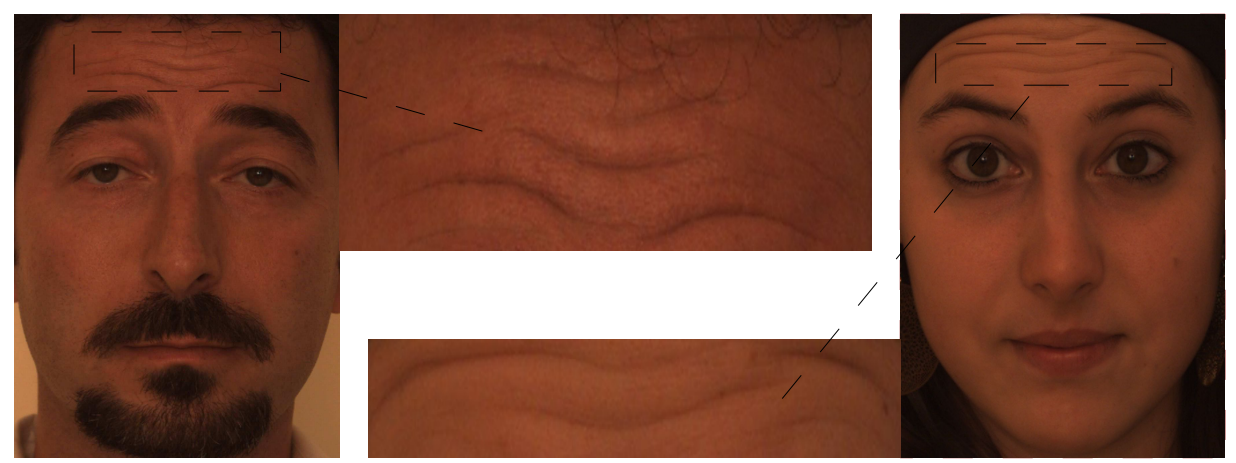

Fig. 4. Sample of forehead image. The dash lines show the portion of forehead image cropped from the original facial image. The image source was from Bosphorus dataset [19]. 


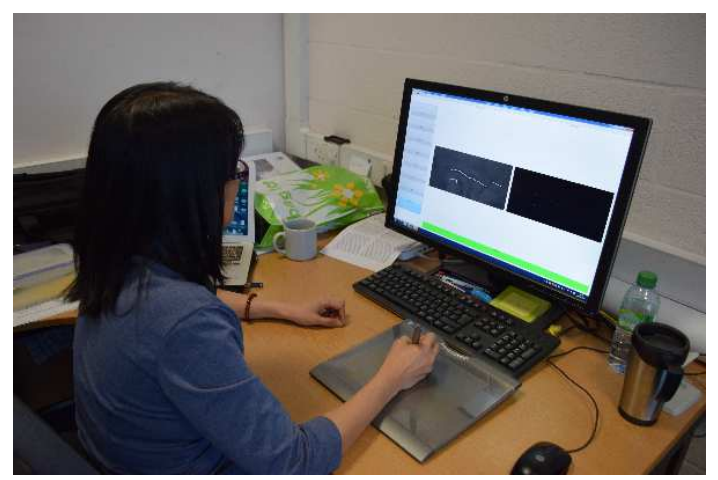

Fig. 5. Coder is doing wrinkle annotation in Matlab.

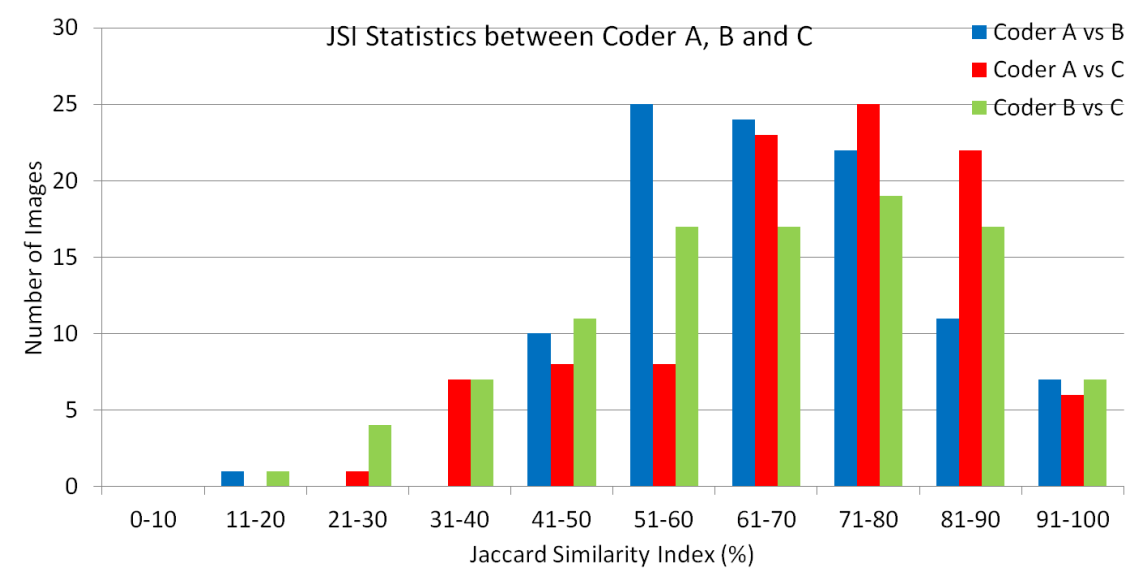

Fig. 6. JSI between coders: A and B, A and C, B and C.

with hand-labelling as the ground truth. Three coders have been instructed to do the annotation in Matlab under a controlled environment as illustrated in Fig. 5 (Microsoft Windows 7 Enterprise 64-bit SP1, Intel Core i7-3770 CPU @ 3.40GHZ, 8.0GB RAM, NVIDIA Quadro 200, lab with similar lighting condition). They were instructed how to use the annotation tool, annotating on the interior center line of the wrinkle according to what they see as a wrinkle. Center line of a wrinkle means the deepest wrinkle area. The reason for this is to minimize the factors such as lighting, screen size and the noise interference while annotating the wrinkles. In this work, we are interested in horizontal wrinkles as most forehead wrinkles are horizontal. Wrinkles are annotated only within those less than $45^{\circ}$. One pixel line was used for each manual annotation. Fig. 1(b) illustrates the mask of manual annotation. 


\subsection{Manual Annotation}

In the first experiment, we validated the reliability of manual annotation among coders. Coders were given the forehead images in the computer and they performed the wrinkle annotation by saving the wrinkle mask in the logical format of Matlab. Then, the annotated masks were used for calculating the JSI between coders as shown in Fig. 6. In this work, we used JSI threshold equals to $40 \%$ as a benchmark of reliability [21]. Any overlapping above this threshold is considered reliable. For the coder A and B, almost $99 \%$ of the JSI is above the threshold and the standard deviation (STD) is 14.27; for the coder A and C, $92 \%$ of the JSI is above the threshold and STD is 16.77; and for the coder B and C, $88 \%$ of the JSI is above the threshold and STD is 18.61 . The average reliability of manual annotation between coder A, B and $\mathrm{C}$ is $93 \%$. We noticed that most of the coarse wrinkles were correctly annotated, but not the fine wrinkles. This experiment shows the inconsistency among coders. Fig. 7 shows the samples of manual annotation between three coders. As we noticed that coder B did not annotate certain lines of all images as wrinkles but coder A did annotate it such as img i, ii and iv. Such contradictions resulted in the variations of annotation and hence yielded different JSI results. Moreover, this result demonstrates the difficulty of wrinkle localization and the high technical challenge for automatic wrinkle detection.

\subsection{Performance Assessment against Benchmark Algorithm}

Fig. 8 shows the automatic detection output of coarse wrinkles. Top right is the CLM output with JSI $18.41 \%$, bottom left is the FRF output with JSI $49.23 \%$, and bottom right is the HHF output with JSI $64.14 \%$. HHF increased the true positive rate, but it also generated false wrinkle.

Fig. 9 illustrates the outputs of wrinkle detection for medium wrinkles. Top left is the original image, top right is the CLM output with JSI $12.22 \%$, bottom left is the FRF output with JSI 19.44\%, and bottom right is the HHF output with JSI $48.68 \%$. In this case, the wrinkles are less visible than the image of Fig. 8.

Fig. 10 presents the outputs of fine wrinkle detection. Left is the original image and right is the HHF output with JSI 10.31\%. Other methods failed to detect any wrinkles. The wrinkles in this image are very blurred compared to the previous examples. This is what we meant by low visibility where the coders might have large variations of annotations.

In this experiment, we repeated the CLM and FRF experiments based on our understanding and certain functions were derived from the available sources in [22], [23]. All derived functions used the default parameters. Fig. 11 shows the results of wrinkle detection by using the state-of-the-art methods (CLM and FRF) and the proposed method HHF. As we observed HHF appears to perform better than CLM and FRF. The average of CLM, FRF and HHF are $54.00 \%$, $61.67 \%$ and $75.67 \%$; the STD are 4.36, 8.08 and 7.51, respectively. One reason for this might be that in HHF the directional gradient has greatly smoothed the 


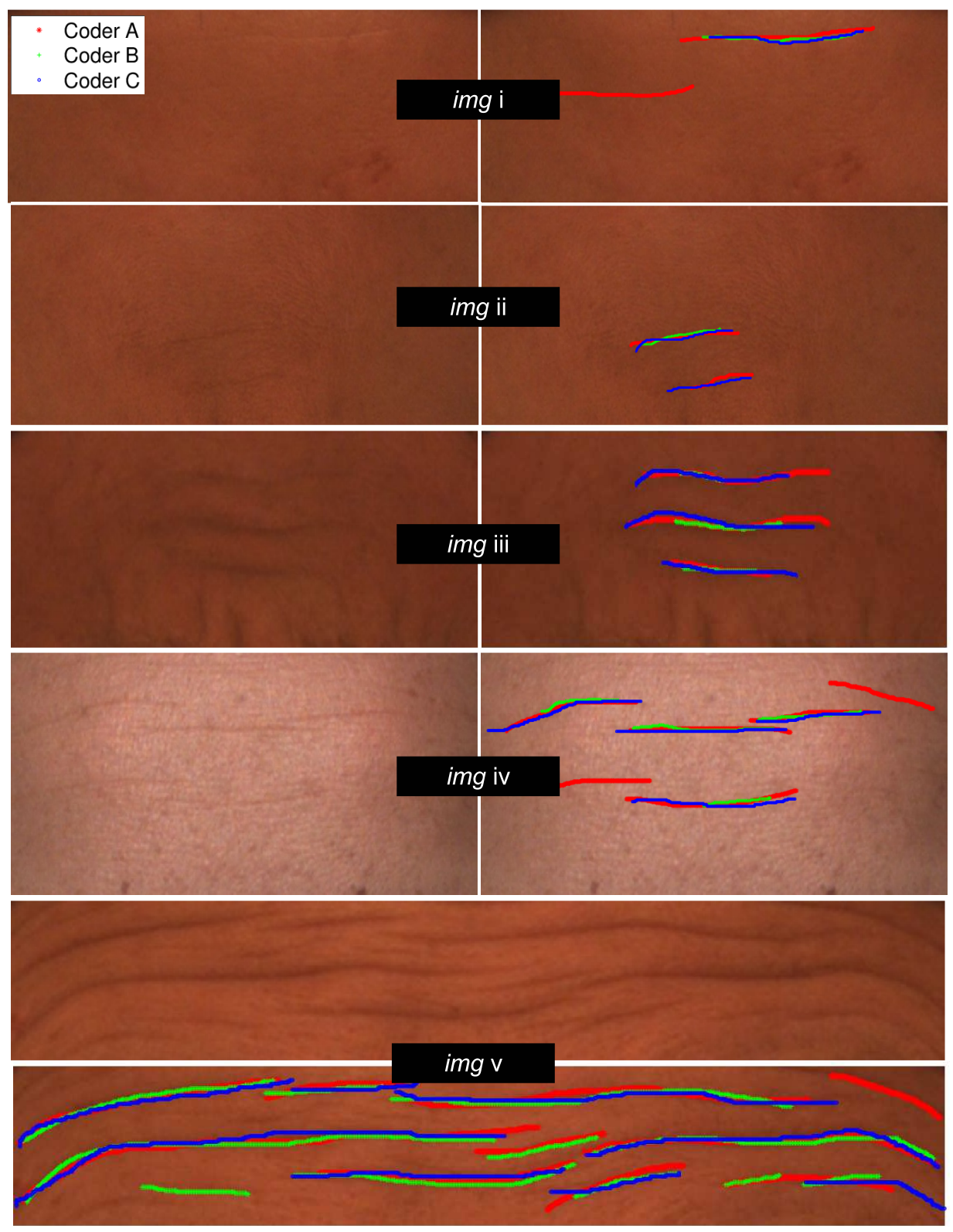

Fig. 7. Samples of Manual Annotation. Each row shows an original image (left) and annotated image of three coders (right). Red line represents coder A, green line is coder $\mathrm{B}$ and blue line is coder C. $i m g$ i, ii, iii, iv, v mean different images.

image and preserved the data of interest. Cula et al. [4] argued that red channel of image and histogram equalization will strengthen the wrinkle image, but the 

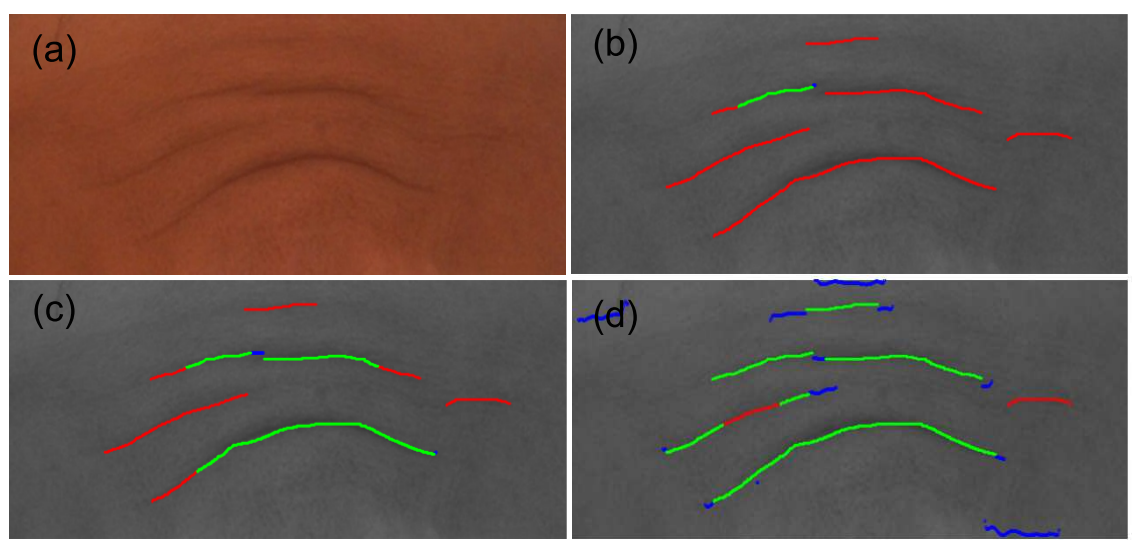

Fig. 8. Automatic detection of coarse wrinkles. (a) Original image. (b), (c) and (d) are the wrinkle detection by CLM, FRF and HHF, respectively. Red: ground truth, green: true positive, blue: false positive.

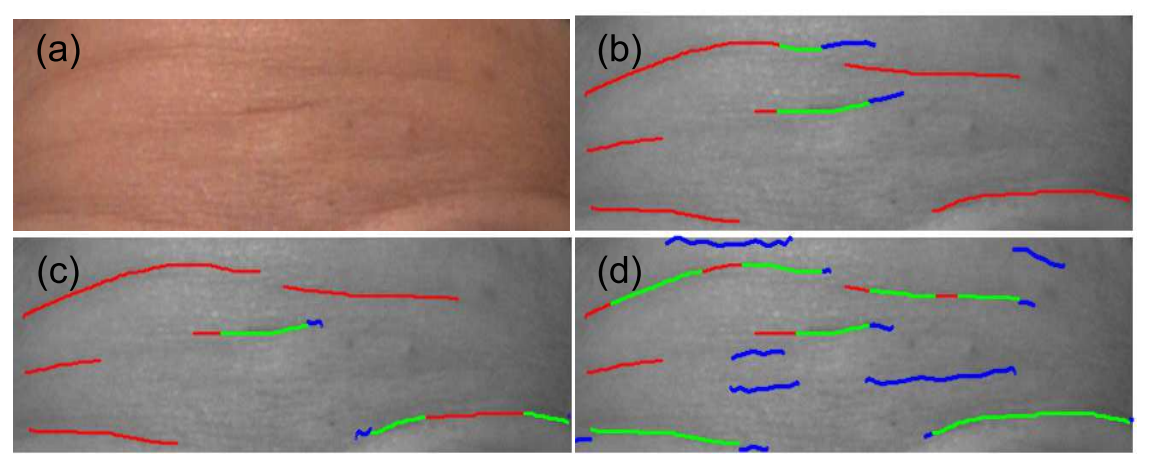

Fig. 9. Automatic detection of medium wrinkles. (a) Original image. (b), (c) and (d) are the wrinkle detection by CLM, FRF and HHF, respectively. Red: ground truth, green: true positive, blue: false positive.
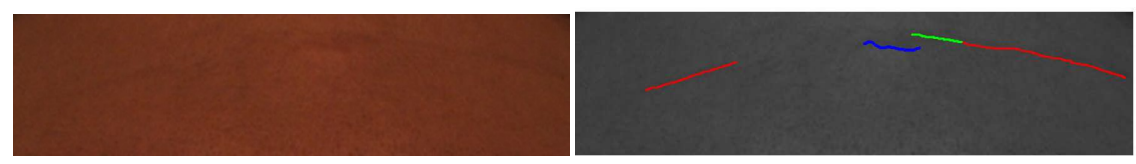

Fig. 10. Automatic detection of fine wrinkles. Left is the original image and right is the HHF output. Red: ground truth, green: true positive, blue: false positive.

CLM result was poor. Histogram equalization is good when the histogram of the image is confined to a particular region, but it is not useful on certain images which have large intensity variations where the histogram covers a large region. 


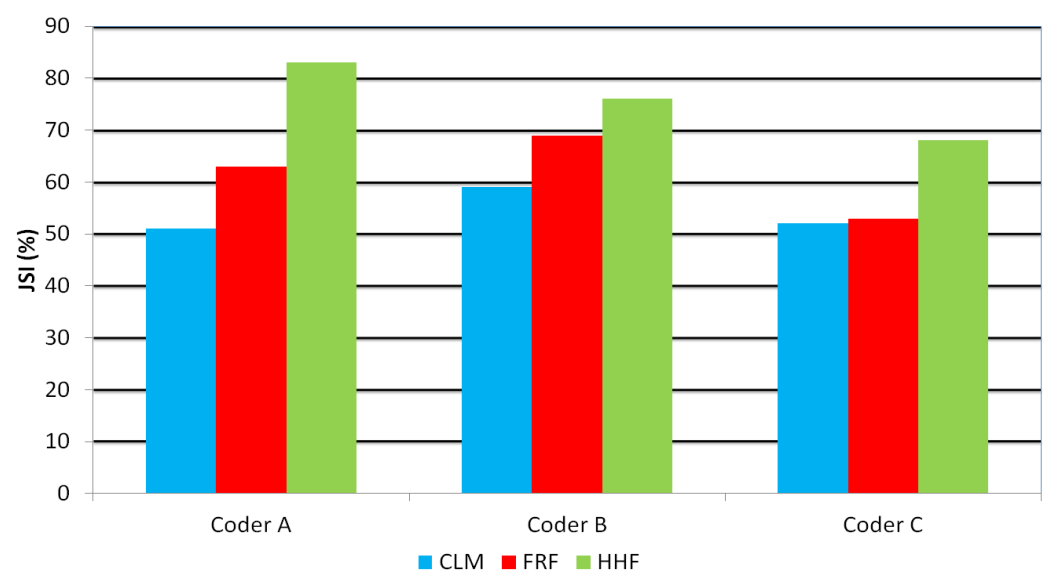

Fig. 11. JSI of automatic wrinkle detection.

This experiment illustrates that the HHF is capable of detecting the wrinkles compared to the conventional edge detection methods. Although HHF is outperformed, further investigation is needed on the issues of over-segmentation and sensitivity to the fine wrinkles. In order to reduce the false alarm, more efforts are needed to improve second order structure of Hessian matrix at each scale. In addition, the distribution of ridge likeliness is another aspect which could be explored. In future work, this experiment will be extended to detect the wrinkles on the face especially in the area of crows feet and mouth corner. Moreover, the relationship between the detected wrinkles and facial aging will be investigated.

\section{Conclusion}

This paper contributes to the groundwork of automated wrinkle detection using a new approach, which will bring advancements to the research in facial ageing, cosmetics and soft biometrics. We described a novel method hybrid Hessian filter (HHF) for wrinkle detection in 2D facial image. It is based on the directional gradient and Hessian matrix. This matrix is computed for all the pixels on the image. The maximum eigenvalues of the Hessian matrix will indicate if a point belongs to a ridge regardless of the ridge orientation. We compared the proposed method with the Cula method (CLM) and Frangi filter (FRF). The experimental results demonstrated that $\mathrm{HHF}$ outperforms state-of-the-art methods with an average JSI of $75.67 \%$. The idea to integrate the directional gradient followed by a second-order filtering outperformed the state-of-the-art methods. Although there is a gap between human observation and automatic wrinkle detection, the proposed HHF significantly increases the number of true detection and wrinkles can be correctly localized at the desired location. 
Acknowledgement. This work was supported by Manchester Metropolitan University's PhD Studentship.

\section{References}

1. Gartstein, V., Shaya, S.A.: Image analysis of facial skin features. In: Application of Optical Instrumentation in Medicine XIV and Picture Archiving and Communication Systems (PACS IV) for Medical Applications, International Society for Optics and Photonics (1986) 284-289

2. Mizukoshi, K., Takahashi, K.: Analysis of the skin surface and inner structure around pores on the face. Skin Research and Technology 20 (2014) 23-29

3. Luebberding, S., Krueger, N., Kerscher, M.: Comparison of validated assessment scales and $3 \mathrm{~d}$ digital fringe projection method to assess lifetime development of wrinkles in men. Skin Research and Technology 20 (2014) 30-36

4. Cula, G.O., Bargo, P.R., Nkengne, A., Kollias, N.: Assessing facial wrinkles: automatic detection and quantification. Skin Research and Technology 19 (2013) e243-e251

5. Kwon, Y., da Vitoria Lobo, N.: Age classification from facial images. Computer Vision and Image Understanding 74 (1999) 1-21

6. Ramanathan, N., Chellappa, R., Biswas, S.: Computational methods for modeling facial aging: A survey. Journal of Visual Languages \& Computing 20 (2009) 131144

7. Fu, Y., Guo, G., Huang, T.: Age synthesis and estimation via faces: A survey. IEEE Transactions on Pattern Analysis and Machine Intelligence 32 (2010) 1955-1976

8. Aznar-Casanova, J., Torro-Alves, N., Fukusima, S.: How much older do you get when a wrinkle appears on your face? modifying age estimates by number of wrinkles. Aging, Neuropsychology, and Cognition 17 (2010) 406-421

9. Choi, Y.H., Tak, Y.S., Rho, S., Hwang, E.: Accurate wrinkle representation scheme for skin age estimation. In: 5th FTRA International Conference on Multimedia and Ubiquitous Engineering (MUE), IEEE (2011) 226-231

10. Huang, Y., Li, Y., Fan, N.: Robust symbolic dual-view facial expression recognition with skin wrinkles: local versus global approach. IEEE Transactions on Multimedia 12 (2010) 536-543

11. Bando, Y., Kuratate, T., Nishita, T.: A simple method for modeling wrinkles on human skin. In: Proceedings of the 10th Pacific Conference on Computer Graphics and Applications, IEEE (2002) 166-175

12. Batool, N., Chellappa, R.: Modeling and detection of wrinkles in aging human faces using marked point processes. In: Computer Vision-ECCV 2012. Workshops and Demonstrations, Springer (2012) 178-188

13. Kwon, Y., da Vitoria Lobo, N.: Age classification from facial images. In: Proceedings of the IEEE Computer Society Conference on Computer Vision and Pattern Recognition, IEEE (1994) 762-767

14. Hayashi, J.i., Yasumoto, M., Ito, H., Koshimizu, H.: Age and gender estimation based on wrinkle texture and color of facial images. In: Proceedings of the 16th International Conference on Pattern Recognition. Volume 1., IEEE (2002) 405-408

15. Frangi, A.F.: Three-dimensional model-based analysis of vascular and cardiac images. Phd thesis, University Medical Center Utrecht (2001)

16. Sofka, M., Stewart, C.V.: Retinal vessel centerline extraction using multiscale matched filters, confidence and edge measures. IEEE Transactions on Medical Imaging 25 (2006) 1531-1546 
17. Martinez-Perez, M.E., Hughes, A.D., Thom, S.A., Bharath, A.A., Parker, K.H.: Segmentation of blood vessels from red-free and fluorescein retinal images. Medical image analysis 11 (2007) 47-61

18. Liu, S., Silverman, M.: A practical guide to biometric security technology. IT Professional 3 (2001) 27-32

19. Savran, A., Sankur, B., Taha Bilge, M.: Regression-based intensity estimation of facial action units. Image and Vision Computing 30 (2012) 774-784

20. Jaccard, P.: Distribution de la flore alpine dans le bassin des drouces et dans quelques regions voisines. Bulletin de la Socit Vaudoise des Sciences Naturelles 37 (1901) 241-272

21. Real, R.: Tables of significant values of jaccard's index of similarity. Miscel- lània Zoològica 22 (1999) 29-40

22. Kovesi, P.: Matlab and octave functions for computer vision and image processing. (http://www.csse.uwa.edu.au/ $\sim \mathrm{pk} /$ ) Accessed on March 2014.

23. Kroon, D.J.: Hessian based frangi vesselness filter. (Matlab Central, http://www.mathworks.co.uk/) Accessed on January 2014. 